\title{
Characteristic of Parkinson's disease with severe COVID-19: a study of 10 cases from Wuhan
}

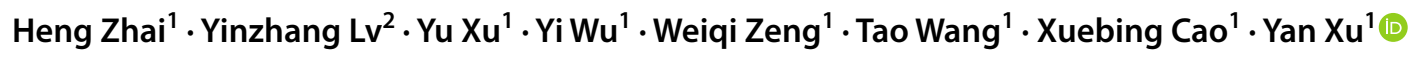

Received: 4 August 2020 / Accepted: 25 November 2020 / Published online: 3 January 2021

(c) The Author(s) 2021

\begin{abstract}
Information about Parkinson's disease (PD) patients with severe COVID-19 is scarce. We aimed to analyze the clinical characteristics, outcomes, and risk factors affecting the prognosis of PD patients with severe COVID-19 infection. Clinical data of severe COVID-19 patients admitted at the Union Hospital, Wuhan between 28th January and 29th February 2020 were collected and analyzed. 10 patients $(1.96 \%)$ had a medical history of PD with a mean (SD) age of $72.10( \pm 11.46)$ years. The clinical characteristics and outcomes of severe COVID-19 with and without PD patients were then compared. There was no significant difference in overall mortality between the PD and non-PD patients with severe COVID-19 $(p>0.05)$. In PD patients with severe COVID-19, the proportion of patients with critical type, disturbance of consciousness, incidence of complications, white blood cells count and neutrophils counts on admission seem higher in the non-survivors. PD patients with older age, longer PD duration, and late stage PD may be highly susceptible to critical COVID-19 infection and bad outcome. The PD patients with consciousness disorders and complications that progressed rapidly are at increased risk of death.
\end{abstract}

Keywords Parkinson's disease $\cdot$ COVID-19 $\cdot$ SARS-CoV-2 $\cdot$ Clinical characteristics $\cdot$ Clinical outcomes

\begin{tabular}{llll}
\multicolumn{2}{l}{ Abbreviations } & BUN & Blood urea nitrogen \\
PD & Parkinson's disease & Cr & Creatinine \\
COVID-19 & Coronavirus disease 2019 & CK & Creatine kinase \\
SARS-CoV-2 & Severe acute respiratory syndrome corona- & LDH & Lactate dehydrogenase \\
& virus 2 & CRP & C-reactive protein \\
ALT & Serum alanine aminotransferase & ICU & Intensive care unit \\
AST & Aspartate aminotransferase & ARDS & Acute respiratory distress syndrome \\
& & DIC & Diffusive intravascular coagulation
\end{tabular}

Heng Zhai and Yinzhang Lv contributed equally to this work.

Supplementary Information The online version contains supplementary material available at https://doi.org/10.1007/s0070 2-020-02283-y.

Tao Wang

wangtaowh@hust.edu.cn

$\triangle$ Xuebing Cao

caoxuebing@126.com

$\triangle$ Yan Xu

xuyanwxf@126.com

1 Department of Neurology, Union Hospital, Tongji Medical College, Huazhong University of Science and Technology, Wuhan 430022, China

2 Department of Radiology, Tongji Hospital, Tongji Medical College, Huazhong University of Science and Technology, Wuhan, China

\section{Introduction}

Coronavirus disease 2019 (COVID-19) is an acute infectious respiratory disease caused by severe acute respiratory syndrome coronavirus 2 (SARS-CoV-2). It was first reported in Wuhan, China in December 2019 and quickly spread across the world within few months (Zhu et al. 2020). This virus has poorer outcomes and higher mortality rates in older people and those with comorbidities or chronic diseases. This being the case, patients suffering from Parkinson's Disease (PD) are also likely to be susceptible to COVID-19 infection and evolve to a severe condition, and possible death, because of their old age and low immunity (Borges et al. 2020; Helmich et al. 2020; Shahid et al. 2020). 
To date, there are only few clinical studies on PD patients with active COVID-19 infection. It was showed that PD with older age and longer disease duration are particularly susceptible to COVID-19 with a high mortality (Antonini et al. 2020; Hainque et al. 2020). However, it is not entirely clear whether the mortality of PD is higher than that of the general population. Moreover, the risk factors for death and whether PD itself has a negative effect on COVID-19 patients have not been accurately defined.

The study aims to determine the clinical manifestations, and outcomes of PD patients with severe COVID-19 and to further explore the risk factors associated with in-hospital mortality of PD patients in the early stage of the epidemic. We hope that this work will be valuable for helping clinicians identify patients with poor prognosis at an early stage by catching some of the alarming clinical characteristics presented by PD patients with COVID-19, and guide effective management for PD patients.

As such, clinical data were collected from severe and critically ill COVID-19 patients in West Branch of Union hospital in Wuhan at the peak of COVID-19 outbreak. The clinical characteristics and outcomes of severe COVID-19 with and without PD as well as survivors and non-survivors of PD were then analyzed.

\section{Methods}

\section{Study design and participants}

This was a single-center, retrospective, and observational study. It was approved by the Ethics committee of Tongji Medical College of Huazhong University of Science and Technology. The study also complied with the Declaration of Helsinki (World Medical Association Declaration of Helsinki Ethical Principles for Medical Research Involving Human Subjects 2013). Clinical data of patients positive for COVID-19 in the West Branch of Union Hospital in Wuhan were collected between 28th January and 29th February 2020 for analysis. This was a designated hospital for the severely ill COVID-19 patients. All patients positive for COVID-19 were diagnosed according to the World Health Organization interim guidelines (WHO. Clinical management of severe acute respiratory infection when novel coronavirus (2019-nCoV) infection is suspected: interim guidance, 2020). Oral consent was obtained directly from the patients or the patients' close relatives prior to data collection.

\section{Data collection}

Demographic data (age, sex, and date of COVID-19 onset) as well as data on the clinical signs and symptoms, medical history, laboratory findings, treatment used, and outcomes were collected from the hospital's electronic medical records. The date of COVID-19 onset was defined as the day when the symptoms were first noticed. The diagnosis and severity of COVID-19 was determined according to the guidelines outlined in the diagnosis and treatment protocol for novel coronavirus (2019-nCoV) disease (Trial Version 7) compiled by the Chinese National Health Commission. The data were then reviewed by a team of trained physicians. The clinical symptoms and treatment measures of PD patients were also reviewed and confirmed by two trained neurologists.

The treatment measures of COVID-19 patients were collected and included. The patients had at least received antiviral agents, antibiotics, immune regulators, oxygen inhalation and mechanical ventilation. $\mathrm{H}-\mathrm{Y}$ stage, duration and antiparkinsonian drugs were collected for patients with PD. The length of hospital stays, course of illness and outcome (discharge or death) were also recorded. COVID19 patients were discharged from the hospital, when they had attained the normal body temperature, remission of clinical symptoms, and resolved lung inflammation proved by a lung computed tomography. The patients displayed at least two consecutive negative results of real-time reverse transcriptase polymerase chain reaction detection for SARS-CoV-2 nucleic acid done at an interval of $24 \mathrm{~h}$.

Laboratory findings on admission including blood cell count, serum alanine aminotransferase (ALT), aspartate aminotransferase (AST), blood urea nitrogen (BUN), creatinine $(\mathrm{Cr})$, creatine kinase $(\mathrm{CK})$, lactate dehydrogenase (LDH), C-reactive protein (CRP), blood glucose, electrolyte, and D-dimer levels were collected.

\section{Statistical analysis}

All statistical analyses were performed using the SPSS software version 23.0. Normality of the data was tested using the Shapiro-Wilk test. Normally distributed continuous variables were presented as means and standard deviations while non-normally distributed continuous variables were presented using median and interquartile range (IQR) values. Categorical variables were expressed as counts and percentages. Means for the normally distributed continuous variables were compared using the independent group $t$-tests while the non-normally distributed data was compared using the Mann-Whitney test. Categorical variables were compared using the unpaired Wilcoxon rank sum test. Their proportions were compared using the $\chi^{2}$ tests. $p$ values less than 0.05 indicated that there were significant differences between groups. 


\section{Results}

\section{Incidence, demographics, and clinical characteristics of PD patients with severe COVID-19 infection}

Between 28th January and 29th February 2020, 510 patients were hospitalized at West Branch of Wuhan Union Hospital and confirmed to severe and critically ill COVID-19. The patients had a median age of 59.5 years (IQR 46-68 years). The most common pre-existing comorbidities among the patients were hypertension (32.35\%), diabetes (19.77\%), cardiovascular diseases $(9.59 \%)$ and cerebrovascular disease $(2.35 \%)$. Patients with hypertension and diabetes had a median age of 68 years (IQR 61-74 years) and a mean (SD) age of 65.87 ( \pm 11.86$)$ years; while, those with cardiovascular disease and cerebrovascular disease had a median age of 67 years (IQR 62-76.5 years) and 81 years (IQR $64.25-83.75$ years), respectively. Only 10 patients (1.96\%) had a medical history of PD. These patients were aged between 56 and 89 years, with a mean (SD) age of 72.10 $( \pm 11.46)$ years (Tables 1,3). Data of 286 patients with the same age range and no history of PD were collected to avoid statistical bias caused by age (Table 3 ). The 286 patients had a median age of 66 years (IQR 62-71 years). There was no significant difference between the age of patients with PD and patients with other comorbidities $(p>0.05)$ (Table 1).

The general information, clinical characteristics, treatment, and outcomes of COVID-19 patients with PD are shown in Table 2. All PD patients were either severe or critically ill on admission. Among them, three patients had hypertension, two had diabetes mellitus, four had cerebrovascular disease, and two had cardiovascular disease. The symptoms of PD patients with COVID-19 included coughing $(n=10 ; 100.00 \%)$, fever $(n=8 ; 80.00 \%)$, fatigue $(n=7$; $70.00 \%)$, anorexia $(n=6 ; 60.00 \%)$, chest tightness $(n=5$; $50.00 \%)$, shortness of breath $(n=4 ; 40.00 \%)$, dyspnea $(n=3$;

Table 1 Comparison in chronic medical illness of COVID-19 patients

\begin{tabular}{llll}
\hline \multicolumn{2}{c}{ No. $(\%)$} & Age (year) & $p$ value \\
\hline Chronic medical & illness & & \\
Hypertension & $165(32.35 \%)$ & $68.00(61.00-74.00)$ & 0.226 \\
DM & $101(19.77 \%)$ & $65.87 \pm 11.86$ & 0.132 \\
CHD & $49(9.59 \%)$ & $67.00(62.00-76.50)$ & 0.422 \\
CVD & $12(2.35 \%)$ & $81.00(64.25-83.75)$ & 0.485 \\
PD & $10(1.96 \%)$ & $72.10 \pm 11.46$ & \\
\hline
\end{tabular}

COVID-19 Coronavirus disease 2019, PD Parkinson's disease, DM diabetes mellitus, $C V D$ cerebrovascular disease, $C H D$ coronary heart disease, No. number

$p$ value indicate differences between age of patients with hypertension, DM, CHD or CVD and patients with PD

$p<0.05$ was considered statistically significant
$30.00 \%)$, myalgia $(n=3 ; 30.00 \%)$, diarrhea $(n=1 ; 10.00 \%)$ and nausea $(n=1 ; 10.00 \%)$ (Table 3$)$. Three patients $(30 \%)$ had rapid deterioration and died soon after admission.

COVID-19 patients with and without PD were given the same treatment strategies including antiviral drugs such as abidor, antibiotics, expectorants, and immune regulators such as thymosin. All of the seven remaining PD patients were on antiparkinsonian medication during their hospitalization. Moreover, all of the $10 \mathrm{PD}$ patients required oxygen supplementation; five had a nasal cannula, four had a facial mask and one was under a mechanical ventilation therapy (Table 2). In PD patients with severe COVID-19, the median hospital stay was 12.00 [IQR 5.25-24.50] days, and the median duration of COVID-19 disease was 30 [IQR 19-51] days (Table 3).

\section{Comparison of clinical characteristics and outcomes between severe COVID-19 patients with and without PD}

Table 3 shows the clinical characteristics and outcomes of severe COVID-19 patients with and without PD. This comparison comprised 296 COVID-19 patients (286 without PD and 10 with PD). Majority of the patients were 70 years old or younger. All patients had either severe or critical illness. Among them, 46.62, 27.27, and $16.84 \%$ of the patients had hypertension, diabetes, and cardiac disease, respectively. The proportion of patients with cerebrovascular diseases among the PD patients with COVID-19 was $40.00 \%$. This was significantly higher than that of COVID-19 patients without PD (2.8\%).

The proportion of PD patients with cough $(100.00 \%$, $n=10)$ and anorexia $(60.00 \%, n=6)$ was significantly higher than that of patients without PD $(65.38 \%, n=187$ and $19.23 \%, n=55$, respectively) $(p<0.05)$. There were no significant differences in lengths of hospital stay and duration of disease between patients with and without PD [12.00 (5.25-24.50) vs. 13 (7-18) days and 30 (19-51) vs. 25 (18-31) days, respectively] $(p>0.05)$. The overall mortality of COVID-19 patients aged between 56 and 89 years was $40.20 \%$. In the same line, the mortality was $30.00 \%$ and $40.56 \%$ in patients with and without PD, respectively. However, this difference was not significant $(p>0.05)$.

\section{Summary of clinical characteristics and laboratory findings of survivors and non-survivors of PD patients with severe COVID-19}

The summary of clinical data of PD patients with severe COVID-19 are shown in Table 4. The median duration of COVID-19 disease of the non-survivors was 13.00 [3.00-21.00] days, and that of the survivors was 43.29 (16.86) days. Further analysis of the disease characteristics 


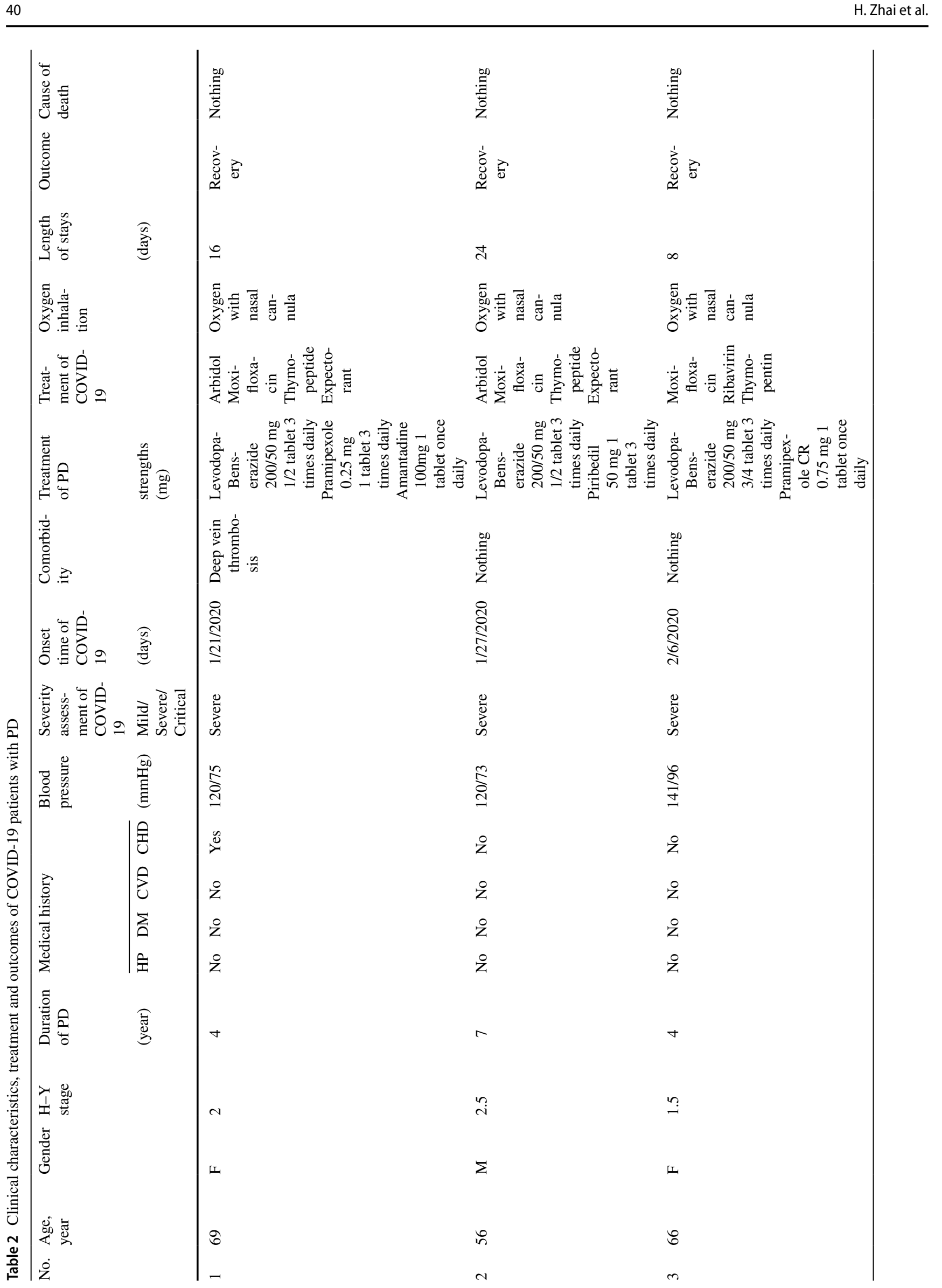

些 Springer 
Characteristic of Parkinson's disease with severe COVID-19: a study of 10 cases from Wuhan

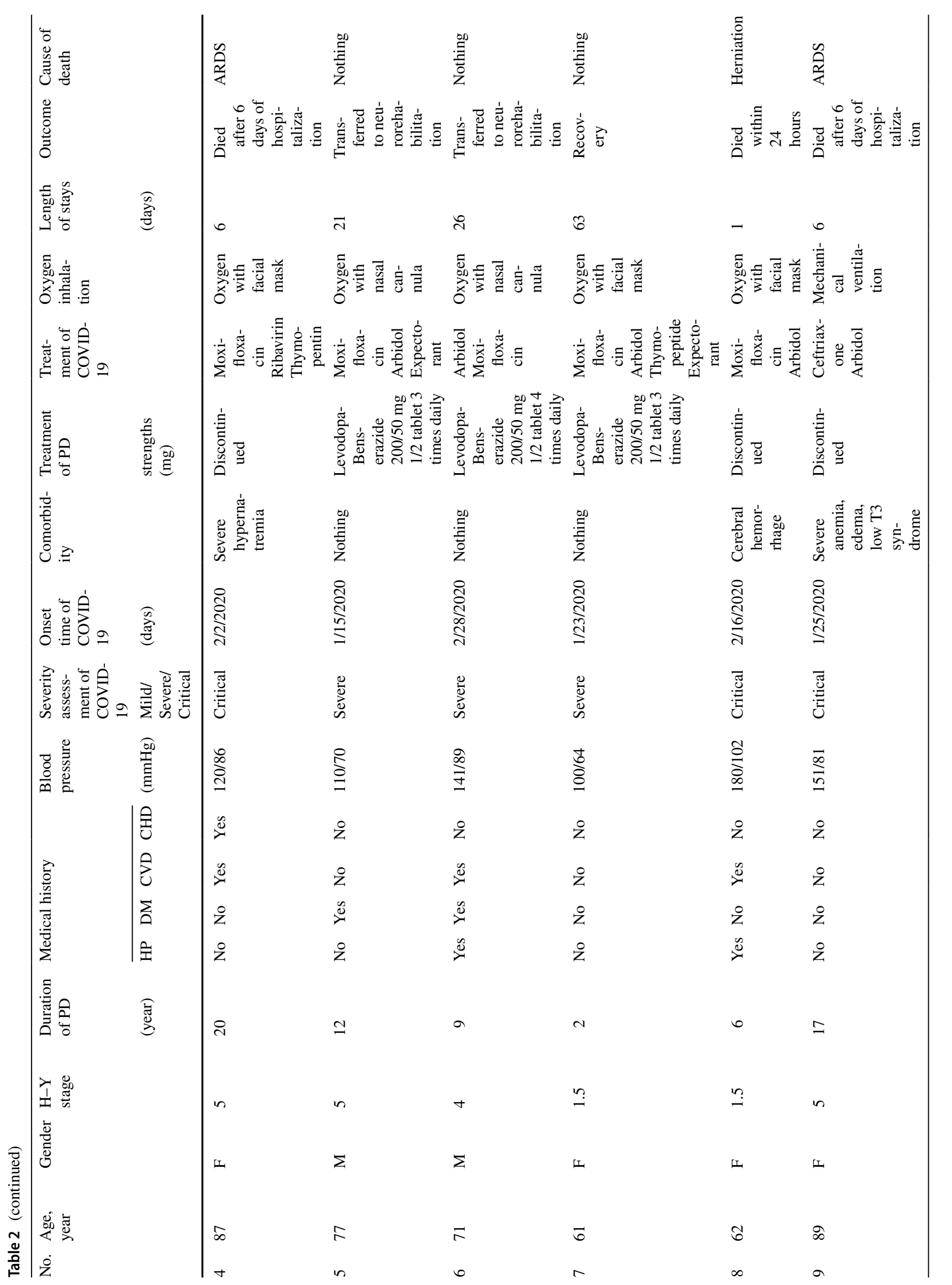

Springer 


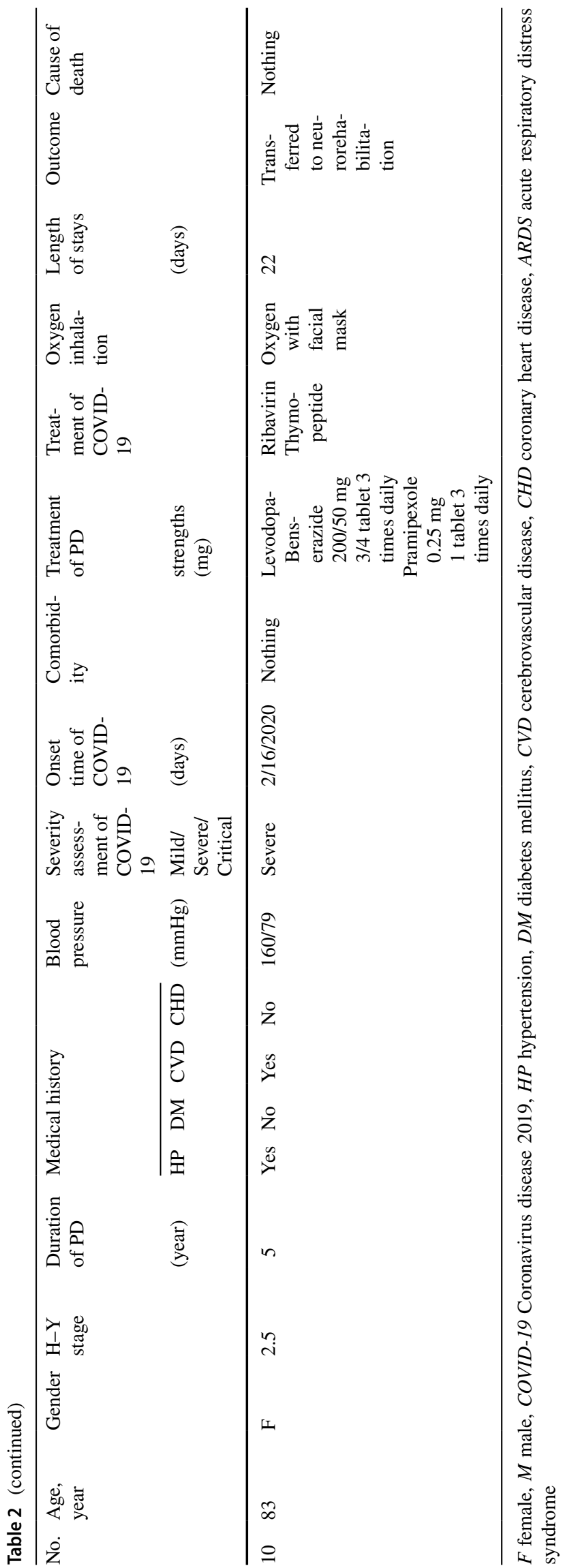

of PD patients revealed that seven of them belonged to the non-tremor dominant type. In addition, eight had abnormal mood. Median age of PD survivors and non-survivors was 67.50 years (IQR 62.25-75.50 years) and 87.00 years (IQR 62.00-89.00 years), respectively.

In chronic comorbidities, $28.57 \%$ of the survivors and $66.7 \%$ of the non-survivors have history of cerebrovascular disease. Based on the Hoehn and Yahr scale (H\&Y), $28 \%$ of the survivors and $67 \%$ of the non-survivors were in $\mathrm{H} \& \mathrm{Y}$ stage $>3$, and two of three non-survivors was in $\mathrm{H} \& \mathrm{Y}$ stage 5. Among the survivors, only one patient (14\%) had duration of PD over ten years, while two $(66 \%)$ of three non-survivors had more than ten years of PD course. One $(14 \%)$ of the survivors and two $(66 \%)$ of non-survivors had cognitive impairment, mental disorder and dysphagia. The three non-survivors were all critical illness. The proportion of patients with disturbance of consciousness on admission in the non-survivors (100\%) seemed higher than that of the survivors (14.29\%). Similarly, the non-survivors seemed to have a higher white blood cell count and neutrophil count.

Moreover, all of the non-survivors and one of survivors had incidence of complications. Among three PD patients (30\%) who died, one 62-year-old patient died from cerebral hernia that was secondary to cerebral hemorrhage within $24 \mathrm{~h}$ after admission. The other two non-survivors who were aged above 85 years died from ARDS that rapidly deteriorated into severe complications, with one non-survivors had severe hypernatremia, while the other had severe edema. Only one patient among the seven survivors developed venous thrombosis of lower extremity.

Regarding the antiparkinsonian drugs applied during hospitalization, the three non-survivors did not receive antiparkinsonian drugs anymore, and the seven survivors continued using antiparkinsonian drugs. Levodopa was used by all survivors.

\section{Discussion}

This was a single-centered retrospective study that described the incidence, clinical characteristics, outcomes and relative risk factors of $\mathrm{PD}$ patients with either severe or critical COVID-19. Early diagnosis and timely treatment to reduce mortality are of crucial importance. PD patients may gradually worsen the motor or non-motor symptoms in the early stages of COVID-19 (Hainque et al. 2020). In our study, the proportion of PD patients with anorexia was higher than those without PD. The mechanism of appetite regulation among PD patients is complex. It involves a decrease in anorexigenic hormones and dopaminergic reward mechanisms, which in turn leads to anhedonia and influences food intake habits (Cersosimo et al. 2018; Der-Avakian et al. 2012). SARS-CoV-2 was found to enter the brain via olfactory 
Table 3 Demographic, clinical characteristics and outcomes of COVID-19 patients with and without PD

\begin{tabular}{|c|c|c|c|c|}
\hline & Total $(n=296)$ & COVID-19 with PD $(n=10)$ & $\begin{array}{l}\text { COVID-19 without PD } \\
(n=286)\end{array}$ & $p$ value \\
\hline Age (year), median (IQR) & $66.00(62.00-71.00)$ & $70.00(61.75-84.00)$ & $66.00(62.00-71.00)$ & 0.229 \\
\hline$\geq 70$ year, No. $(\%)$ & $106(35.81 \%)$ & $5(50.00 \%)$ & $101(35.31 \%)$ & 0.338 \\
\hline$<70$ year, No. $(\%)$ & $190(64.19 \%)$ & $5(50.00 \%)$ & $185(64.69 \%)$ & \\
\hline \multicolumn{5}{|l|}{ Gender, No. (\%) } \\
\hline Male & $150(50.68 \%)$ & $3(30.00 \%)$ & $147(51.40 \%)$ & 0.213 \\
\hline Female & $146(49.32 \%)$ & $7(70.00 \%)$ & $139(48.60 \%)$ & \\
\hline \multicolumn{5}{|c|}{ Chronic medical illness, No. (\%) } \\
\hline Hypertension & $138(46.62 \%)$ & $3(30.00 \%)$ & $135(47.20 \%)$ & 0.349 \\
\hline Diabetes mellitus & $81(27.27 \%)$ & $2(20.00 \%)$ & $79(27.62 \%)$ & 0.773 \\
\hline Cerebral vascular disease & $12(3.04 \%)$ & $4(40.00 \%)$ & $8(2.80 \%)$ & 0 \\
\hline Malignant neoplasm & $23(7.74 \%)$ & $0(0.00 \%)$ & $23(8.04 \%)$ & 1 \\
\hline Heart disease & $50(16.84 \%)$ & $2(20.00 \%)$ & $48(16.78 \%)$ & 1 \\
\hline Chronic kidney disease & $7(2.36 \%)$ & $0(0.00 \%)$ & $7(2.45 \%)$ & 1 \\
\hline COPD & $6(2.02 \%)$ & $0(0.00 \%)$ & $6(2.10 \%)$ & 1 \\
\hline Tuberculosis & $2(0.67 \%)$ & $0(0.00 \%)$ & $2(0.70 \%)$ & 1 \\
\hline \multicolumn{5}{|c|}{ Severity assessment of COVID-19, No. (\%) } \\
\hline Severe & $175(59.12 \%)$ & $6(60.00 \%)$ & $169(59.09 \%)$ & 1 \\
\hline Critical & $121(40.88 \%)$ & $4(40.00 \%)$ & $117(40.91 \%)$ & \\
\hline \multicolumn{5}{|c|}{ Symptoms of COVID-19, No. (\%) } \\
\hline Fever & $255(86.15 \%)$ & $8(80.00 \%)$ & $247(86.36 \%)$ & 0.634 \\
\hline Cough & $197(66.55 \%)$ & $10(100.00 \%)$ & $187(65.38 \%)$ & 0.034 \\
\hline Fatigue & $161(54.39 \%)$ & $7(70.00 \%)$ & $154(53.85 \%)$ & 0.305 \\
\hline Chest tightness & $121(40.88 \%)$ & $5(50.00 \%)$ & $116(40.56 \%)$ & 0.745 \\
\hline Shortness of breath & $140(47.30 \%)$ & $4(40.00 \%)$ & $136(47.55 \%)$ & 0.753 \\
\hline Dyspnea & $102(34.46 \%)$ & $3(30.00 \%)$ & $99(34.62 \%)$ & 1 \\
\hline Myalgia & $70(23.65 \%)$ & $3(30.00 \%)$ & $67(23.43 \%)$ & 0.705 \\
\hline Anorexia & $61(20.61 \%)$ & $6(60.00 \%)$ & $55(19.23 \%)$ & 0.006 \\
\hline Diarrhea & $42(14.19 \%)$ & $1(10.00 \%)$ & $41(14.34 \%)$ & 1 \\
\hline Nausea & $25(8.45 \%)$ & $1(10.00 \%)$ & $24(8.39 \%)$ & 0.592 \\
\hline Length of stay (days) & $13.00(7.00-18.75)$ & $12.00(5.25-24.50)$ & $13.00(7.00-18.00)$ & 0.804 \\
\hline Duration (days) & $26.00(18.00-31.00)$ & $30.00(19.00-51.00)$ & $25.00(18.00-31.00)$ & 0.121 \\
\hline \multicolumn{5}{|l|}{ Clinical outcome, No. (\%) } \\
\hline Survived & $177(59.80 \%)$ & $7(70.00 \%)$ & $170(59.44 \%)$ & 0.745 \\
\hline Died & $119(40.20 \%)$ & $3(30.00 \%)$ & $116(40.56 \%)$ & \\
\hline
\end{tabular}

COVID-19 Coronavirus disease 2019, PD Parkinson's disease, COPD chronic obstructive pulmonary disease

$p$ value indicate differences between COVID-19 patients with and without PD

$p<0.05$ was considered statistically significant

bulbs in a mouse model. This explained the potential pathogenic mechanism of taste and smell disorders in patients with COVID-19 infection (Netland et al. 2008; Xu et al. 2020). Early and accurate diagnosis of COVID-19 in PD patients may be challenging (Hainque et al. 2020). As such, the focus should be given to poor appetite in PD patients during the COVID-19 epidemic.

Common comorbidities among patients with COVID-19 with and without PD were hypertension, diabetes mellitus, and coronary heart disease. This was consistent with those reported by Huang and colleagues (Huang et al. 2020). However, the proportion of patients with cerebrovascular disease was higher among those with PD than among those without PD, and the PD non-survivors seemed have higher incidence of cerebrovascular disease. Previous studies found that patients with stroke were more likely to have PD, and PD increases stroke risk and influences poststroke outcomes (Huang et al. 2019). SARS-CoV patients with cerebrovascular disease have a higher risk of critical illness and multiple organ dysfunction (Aggarwal et al. 
Table 4 Clinical characteristics and laboratory findings between survivors and non-survivors with COVID-19 and PD

\begin{tabular}{|c|c|c|c|}
\hline & \multicolumn{3}{|l|}{ COVID-19 with PD } \\
\hline & Total $(n=10)$ & Survivors $(n=7)$ & Non-survivors $(n=3)$ \\
\hline Duration (days) & $34.00 \pm 20.76$ & $43.29 \pm 16.86$ & $13.00(3.00-21.00)$ \\
\hline Age (year), median (IQR) & $70.00(61.75-84.00)$ & $67.50(62.25-75.50)$ & $87.00(62.00-89.00)$ \\
\hline$\geq 70$ year, No. $(\%)$ & $5(50.00 \%)$ & $3(42.86 \%)$ & $2(66.67 \%)$ \\
\hline$<70$ year, No. $(\%)$ & $5(50.00 \%)$ & $4(57.14 \%)$ & $1(33.33 \%)$ \\
\hline \multicolumn{4}{|l|}{ Gender, No. $(\%)$} \\
\hline Male & $3(30.00 \%)$ & $3(42.86 \%)$ & $0(0.00 \%)$ \\
\hline Female & $7(70.00 \%)$ & $4(57.14 \%)$ & $3(100.00 \%)$ \\
\hline \multicolumn{4}{|l|}{ Comorbidities, No. (\%) } \\
\hline Hypertension & $3(30.00 \%)$ & $2(28.57 \%)$ & $1(33.33 \%)$ \\
\hline Diabetes mellitus & $2(20.00 \%)$ & $2(28.57 \%)$ & $0(0.00 \%)$ \\
\hline Cerebrovascular disease & $4(40.00 \%)$ & $2(28.57 \%)$ & $2(66.67 \%)$ \\
\hline Coronary heart disease & $2(20.00 \%)$ & $1(14.29 \%)$ & $1(33.33 \%)$ \\
\hline Chronic kidney disease & $1(10.00 \%)$ & $0(0.00 \%)$ & $1(33.33 \%)$ \\
\hline \multicolumn{4}{|l|}{ Characteristics of PD, No. (\%) } \\
\hline Course $\geq 10$ years & $3(30.00 \%)$ & $1(14.29 \%)$ & $2(66.67 \%)$ \\
\hline 5 years $\leq$ Course $<10$ years & $4(40.00 \%)$ & $3(42.86 \%)$ & $1(33.33 \%)$ \\
\hline Course $<5$ years & $3(30.00 \%)$ & $3(42.86 \%)$ & $0(0.00 \%)$ \\
\hline Non tremor dominant & $7(70.00 \%)$ & $4(57.14 \%)$ & $3(100.00 \%)$ \\
\hline $\mathrm{H}-\mathrm{Y}$ grade $\geq 3$ & $4(40.00 \%)$ & $2(28.57 \%)$ & $2(66.67 \%)$ \\
\hline Abnormal mood & $8(80.00 \%)$ & $5(71.43 \%)$ & $3(100.00 \%)$ \\
\hline Motor complications & $5(50.00 \%)$ & $3(42.86 \%)$ & $2(66.67 \%)$ \\
\hline Cognitive impairment & $3(30.00 \%)$ & $1(14.29 \%)$ & $2(66.67 \%)$ \\
\hline Mental disorder & $3(30.00 \%)$ & $1(14.29 \%)$ & $2(66.67 \%)$ \\
\hline Dysphagia & $3(30.00 \%)$ & $1(14.29 \%)$ & $2(66.67 \%)$ \\
\hline \multicolumn{4}{|l|}{ Severity assessment of COVID-19, No. (\%) } \\
\hline Severe & $7(70.00 \%)$ & $7(100.00 \%)$ & $0(0.00 \%)$ \\
\hline Critical & $3(30.00 \%)$ & $0(0.00 \%)$ & $3(100.00 \%)$ \\
\hline Disturbance of consciousness, No. (\%) & $4(40.00 \%)$ & $1(14.29 \%)$ & $3(100.00 \%)$ \\
\hline Complication, No. (\%) & $4(40.00 \%)$ & $1(14.29 \%)$ & $3(100.00 \%)$ \\
\hline Cerebral hemorrhage & $1(10.00 \%)$ & $0(0.00 \%)$ & $1(33.33 \%)$ \\
\hline ARDS & $2(20.00 \%)$ & $0(0.00 \%)$ & $2(66.67 \%)$ \\
\hline Deep vein thrombosis & $1(10.00 \%)$ & $1(14.29 \%)$ & $0(0.00 \%)$ \\
\hline Severe hypernatremia & $1(10.00 \%)$ & $0(0.00 \%)$ & $1(33.33 \%)$ \\
\hline \multicolumn{4}{|l|}{ Laboratory findings } \\
\hline White blood cell $>10 \times 109 / \mathrm{L}$, No. $(\%)$ & $4(40.00 \%)$ & $1(14.29 \%)$ & $3(100.00 \%)$ \\
\hline Neutrophils >9×109/L, No. (\%) & $4(40.00 \%)$ & $1(14.29 \%)$ & $3(100.00 \%)$ \\
\hline Lymphocyte < 1×109/L, No. (\%) & $6(60.00 \%)$ & $3(42.86 \%)$ & $3(100.00 \%)$ \\
\hline Hemoglobin < 90 g/L, No. (\%) & $1(10.00 \%)$ & $0(0.00 \%)$ & $1(33.33 \%)$ \\
\hline Blood platelet < $125 \times 109 / \mathrm{L}$, No. $(\%)$ & $2(20.00 \%)$ & $0(0.00 \%)$ & $2(66.67 \%)$ \\
\hline C-reactive protein > 8 mg/L, No. (\%) & $8(80.00 \%)$ & $6(85.71 \%)$ & $2(66.67 \%)$ \\
\hline Serum sodium > 145 mmol/L, No. (\%) & $3(30.00 \%)$ & $1(14.29 \%)$ & $2(66.67 \%)$ \\
\hline Blood glucose > $6.1 \mathrm{mmol} / \mathrm{L}$, No. $(\%)$ & $7(70.00 \%)$ & $4(57.14 \%)$ & $3(100.00 \%)$ \\
\hline D-dimer > 0.5 mg/L, No. (\%) & $8(80.00 \%)$ & $5(71.43 \%)$ & $3(100.00 \%)$ \\
\hline Alanine aminotransferase, $\mathrm{U} / \mathrm{L}$ & & $23.00(10.00-52.00)$ & $17.00(4.00-50.00)$ \\
\hline Aspartate aminotransferase, U/L & & $42.29 \pm 30.83$ & $34.00(9.00-38.00)$ \\
\hline Blood urea nitrogen, $\mathrm{mmol} / \mathrm{L}$ & & $6.97(3.53-7.95)$ & $6.36(5.62-16.57)$ \\
\hline Creatinine, $\mu \mathrm{mol} / \mathrm{L}$ & & $56.97 \pm 17.01$ & $82.1(53.8-93.4)$ \\
\hline Creatine kinase, U/L & & $93.00(68.00-156.25)$ & $124.00(22.00-426.00)$ \\
\hline
\end{tabular}


Table 4 (continued)

\begin{tabular}{llll}
\hline & \multicolumn{2}{l}{ COVID-19 with PD } & \\
\cline { 2 - 4 } & Total $(n=10)$ & Survivors $(n=7)$ & Non-survivors $(n=3)$ \\
\hline $\begin{array}{l}\text { Lactate dehydrogenase, U/L } \\
\text { Antiparkinsonian drug treatment, No. (\%) }\end{array}$ & & $292.29 \pm 118.71$ & $265.00(181.00-407.00)$ \\
$\quad$ Levodopa & $7(70.00 \%)$ & $7(100.00 \%)$ & $0(0.00 \%)$ \\
Dopamine receptor agonist & $4(40.00 \%)$ & $4(57.14 \%)$ & $0(0.00 \%)$ \\
Amantadine & $1(10.00 \%)$ & $1(14.29 \%)$ & $0(0.00 \%)$ \\
Discontinuation of antiparkinsonian drug & $3(30.00 \%)$ & $0(0.00 \%)$ & $3(100.00 \%)$ \\
\hline
\end{tabular}

COVID-19 Coronavirus disease 2019, PD Parkinson's disease, NA not available, ARDS acute respiratory distress syndrome

2020). It was speculated that patients with PD and cerebrovascular disease were more susceptible to become severely ill with COVID-19 and progress to death.

Studies on COVID-19 patients requiring services of the intensive care unit (ICU) revealed that their mortality was $61.5 \%$ (Yang et al. 2020a). Our study was conducted in a hospital designated for severely ill COVID-19 patients who were hospitalized with hypoxemia, and thus, included those requiring ICU services and those who did not. The mortality rate of PD patients was $30.00 \%$, while that of non-PD patients was $40.20 \%$. In our study, the high rate of mortality rate of severe COVID-19 patients with PD was in the early stage of the epidemic, and it needs a larger sample size to be confirmed.

Whether various characteristics of PD have different effects on COVID-19 patients is not entirely clear. A community-based case-control study revealed that mildto-moderate COVID-19 occurred independent of age and PD duration (Cilia et al. 2020). Another study indicated that COVID-19 risk, morbidity and mortality in patients with mild-to-moderate PD did not differ from the general population (Fasano et al. 2020). Antonini (Antonini et al. 2020) examined 10 PD patients drawn from two Parkinson Units in Europe and reported that advanced PD patients had a higher COVID-19 mortality rate, which is associated with older age and longer disease duration. In our study, those PD patients who had older age, $\mathrm{H}-\mathrm{Y}$ stage $>3$ and disease course $>10$ years seem more likely to progress to death once infected with severe COVID-19. This conclusion needs to be further confirmed by large sample control studies.

In our study, non-survivors were critically ill and had loss of consciousness on admission. These patients exhibited rapid deterioration than the survivors, which was similar to the previous study that patients in a more critical condition at ICU admission are at increased risk of death. The most common cause of death was acute respiratory distress syndrome (ARDS), and most patients revealed organ function damage in critically ill patients with COVID-19(Yang et al. 2020b).
A total of four PD patients had incidence of complications, three non-survivors died of complications within a week. Two of them died of ARDS. One died of cerebral hemorrhage within $24 \mathrm{~h}$ post admission, and suffered a cerebral infarction few years before the pandemic. Nevertheless, a survivor with deep vein thrombosis of the lower extremity and without a history of cerebral infarction recovered and got discharged after anticoagulant treatment. Patients with COVID-19 are prone to develop coagulation disorders. The imbalance of platelet production and destruction as well as the disorder of the coagulation system increased the risk of bleeding in patients with COVID-19 (Tang et al. 2020). Moreover, the risk of thrombosis was higher in patients with COVID-19 (Li et al. 2020). Herein, 8 PD patients demonstrated increased D-dimer levels. Though no thrombus was found in two patients who died of ARDS, dynamic analysis revealed that one had a gradual increase in D-dimer levels, a gradual decrease in platelet and hemoglobin levels, and severe hypernatremia during the short course of the COVID19 disease, but had no abnormality during admission (Supplementary Table). In some patients, especially in severe and dead patients, the platelet levels are significantly reduced (Guang et al. 2020; Tang et al. 2020; Wang et al. 2020). Hypernatremia is associated with a hypercoagulable state particularly in an immobile patient, and may be common in COVID-19 patients in ICU (Christ-Crain et al. 2020). Thus, platelet count, D-dimer levels, hemoglobin levels and electrolyte levels at the beginning of admission cannot be used as a guide of severity. A single-center cohort study in Japan showed that diffusive intravascular coagulation (DIC) score may be common clinical predictor of mortality with ARDS (Anan et al. 2018). We speculate that the delayed but aggravated coagulation disorders may mediate the fatal ARDS resulting in death of the PD patients with severe COVID-19. Therefore, these laboratory indicators need to be closely monitored after admission to find coagulation disorders as soon as possible, and an anticoagulant was, therefore, administered in time to avoid the disorder.

Neutrophilia and lymphocytopenia were common findings and more significant among severe cases when 
compared to mild cases with COVID-19 (Chen et al. 2020; Gu et al. 2005; Mo et al. 2020). In our study, lymphocytopenia occurred in $42.86 \%$ survivors and $100 \%$ non-survivors with PD, which is in accordance with the previous studies, suggesting that the severity of lymphocytopenia reflects the severity of SARS-CoV-2 infection. Similarly, the white blood cell count and neutrophil count seem higher in the non-survivors in our study, suggesting the indicators are associated with the severity of illness and thus prognosis of patients with PD and severe COVID-19. In addition, our results showed that two non-survivors who died of ARDS presented a gradual decrease in lymphocyte count with a gradual increase in neutrophil count and the survivors lacked these dynamic changes during the course of the COVID-19 disease (Figure s1, Figure s2), which was consistent with the statement that peripheral neutrophilia and lymphocytopenia associated with delayed but exaggerated immune response mediate the fatal ARDS, multi-organ dysfunction resulting in death of COVID-19 patients (Lingeswaran et al. 2020). Hence, dynamic neutrophilia and lymphocytopenia may help in early diagnosis and predicting severity of PD patients with COVID-19.

Sudden cessation of antiparkinsonian drugs leads to aggravation of PD symptoms and even cause malignant syndrome (Grover et al. 2018). In older advanced PD patients with pre-existing dyspnea, it causes rigidity of the respiratory muscle and impairment of the cough reflex thereby leading to increased severity of COVID-19 and death (van Wamelen et al. 2020). Study of the Kings' College group showed that most PD patients required additional levodopa dosing following the infection (Antonini et al. 2020). Another study demonstrated that motor and non-motor symptoms significantly worsened in the COVID-19 group, requiring therapy adjustment in one-third of cases (Cilia et al. 2020). In our study, three PD patients who died did not have symptoms of advanced malignant syndrome such as high fever, rigidity, tremors, and significant elevation of creatine kinase. We speculate that withdrawal of antiparkinsonian drugs in 3 non-survivors may be due to loss of consciousness or loss of the chance to take medicine. Withdrawal of parkinsonian drugs aggravates the symptoms of PD and promotes death. This, however, needs to be studied in larger clinical studies.

Nevertheless, this study was limited by several factors. It was conducted at a single-center hospital and thus the interpretation of the findings could have been limited by the small number of PD patients. We speculate that the low proportion of COVID-19 patients with PD may be due to lack of outdoor exercise and less social contact with others in patients with PD during the periods of COVID-19 prevalence. In the same line, the effect of COVID-19 on the PD symptoms was not analyzed, because the relevant information was not collected. Moreover, the impact of
COVID-19 on PD was not evaluated for long periods of time. As such, the impact of COVID-19 on PD needs to be evaluated with long-term follow-up of survivors.

\section{Conclusion}

PD patients with the older age, longer PD duration and later stage PD seem have a worse prognosis after getting severe COVID-19. COVID-19 severity and presence of complications could greatly affect the prognosis of the PD patients with severe COVID-19. It is necessary to pay special attention to the loss of appetite of PD patients in addition with other COVID-19 symptoms for early and accurate diagnosis of COVID-19. Dynamic monitoring of organ function and coagulation indicator is needed to identify these changes early with prompt management and prevent further complications. Sustainable and optimized antiparkinsonian therapy may be needed to improve the prognosis of PD patients with COVID-19.

Acknowledgments We thank all patients involved in this study. We thank Prof Keqiang Ye (Department of Pathology and Laboratory Medicine, Emory University School of Medicine, Atlanta, GA, United States) for linguistic assistance of this manuscript.

Author contributions YX had full access to all of the data in the study and take responsibility for the integrity of the data and the accuracy of the data analysis. HZ and YL contributed equally to this work and are co-first authors. HZ collected the data, drafted and revised the manuscript. YL analyzed the data. YX, YW and WZ collected the data. YX designed the study, interpreted data and reviewed the manuscript. XC and TW did critical revision of the manuscript. All authors read and approved the final manuscript.

Funding This study did not receive any specific funding.

Data availability The data that support the findings of this study are available from the corresponding author upon reasonable request.

\section{Compliance with ethical standards}

Conflict of interest The authors declare that they have no conflict of interest.

Ethics approval The study was approved by the Ethics committee of Tongji Medical College of Huazhong University of Science and Technology. The authors also confirm that the study was performed in accordance with the ethical standards as laid down in the 1964 Declaration of Helsinki and its later amendments.

Consent to participate Oral consent was obtained directly from the patients or the patients' close relatives prior to data collection.

Consent for publication Authors declare that consent for publication of blinded data was a part of informed consents obtained from participants. 
Open Access This article is licensed under a Creative Commons Attribution 4.0 International License, which permits use, sharing, adaptation, distribution and reproduction in any medium or format, as long as you give appropriate credit to the original author(s) and the source, provide a link to the Creative Commons licence, and indicate if changes were made. The images or other third party material in this article are included in the article's Creative Commons licence, unless indicated otherwise in a credit line to the material. If material is not included in the article's Creative Commons licence and your intended use is not permitted by statutory regulation or exceeds the permitted use, you will need to obtain permission directly from the copyright holder. To view a copy of this licence, visit http://creativecommons.org/licenses/by/4.0/.

\section{References}

Anan K, Kawamura K, Suga M, Ichikado K (2018) Clinical differences between pulmonary and extrapulmonary acute respiratory distress syndrome: a retrospective cohort study of prospectively collected data in Japan. J Thorac Dis 10(10):5796-5803. https:// doi.org/10.21037/jtd.2018.09.73

Antonini A, Leta V, Teo J, Chaudhuri KR (2020) Outcome of Parkinson's disease patients affected by COVID-19. Mov Disord 35(6):905-908. https://doi.org/10.1002/mds.28104

Borges DNI, Cacic N, Abdulazeem HM, von Groote TC, Jayarajah U, Weerasekara I et al (2020) Novel Coronavirus Infection (COVID19) in humans: a scoping review and meta-analysis. J Clin Med 9(4). https://doi.org/10.3390/jcm9040941

Cersosimo MG, Raina GB, Pellene LA, Micheli FE, Calandra CR, Maiola R (2018) Weight loss in Parkinson's Disease: the relationship with motor symptoms and disease progression. Biomed Res Int 2018:9642524. https://doi.org/10.1155/2018/9642524

Chen N, Zhou M, Dong X, Qu J, Gong F, Han Y et al (2020) Epidemiological and clinical characteristics of 99 cases of 2019 novel coronavirus pneumonia in Wuhan, China: a descriptive study. Lancet 395(10223):507-513. https://doi.org/10.1016/S0140 $-6736(20) 30211-7$

Chen G, Di W, Guo W, Cao Y, Huang D, Wang H et al (2020) Clini$\mathrm{cal}$ and immunologic features in severe and moderate forms of Coronavirus Disease 2019. J Clin Invest 130(5):2620-2629. https ://doi.org/10.1172/JCI137244

Christ-Crain M, Hoorn EJ, Sherlock M, Thompson CJ, Wass J (2020) Endocrinology in the time of COVID-19: management of diabetes insipidus and hyponatraemia. Eur J Endocrinol 183(1):G9G15. https://doi.org/10.1530/EJE-20-0338

Cilia R, Bonvegna S, Straccia G, Andreasi NG, Elia AE, Romito LM et al (2020) Effects of COVID-19 on Parkinson's Disease clinical features: a community-based case-control study. Mov Disord 35(8):1287-1292. https://doi.org/10.1002/mds.28170

Der-Avakian A, Markou A (2012) The neurobiology of anhedonia and other reward-related deficits. Trends Neurosci 35(1):68-77. https ://doi.org/10.1016/j.tins.2011.11.005

Fasano A, Cereda E, Barichella M, Cassani E, Ferri V, Zecchinelli AL et al (2020) COVID-19 in Parkinson's Disease patients living in Lombardy Italy. Mov Disord 35(7):1089-1093. https://doi. org/10.1002/mds.28176

Grover S, Sathpathy A, Reddy SC, Mehta S, Sharma N (2018) Parkinsonism-hyperpyrexia syndrome: a case report and review of literature. Indian J Psychiatry 60(4):499-503. https://doi.org/10.4103/ psychiatry.IndianJPsychiatry_113_18

Gu J, Gong E, Zhang B, Zheng J, Gao Z, Zhong Y et al (2005) Multiple organ infection and the pathogenesis of SARS. J Exp Med 202(3):415-424. https://doi.org/10.1084/jem.20050828
Hainque E, Grabli D (2020) Rapid worsening in Parkinson's disease may hide COVID-19 infection. Parkinsonism Relat Disord 75:126-127. https://doi.org/10.1016/j.parkreldis.2020.05.008

Helmich RC, Bloem BR (2020) The impact of the COVID-19 pandemic on Parkinson's disease: hidden sorrows and emerging opportunities. J Parkinsons Dis 10(2):351-354. https://doi. org/10.3233/JPD-202038

Huang YF, Yeh CC, Chou YC, Hu CJ, Cherng YG, Shih CC et al (2019) Stroke in Parkinson's disease. QJM 112(4):269 274. https://doi.org/10.1093/qjmed/hcz015

Huang C, Wang Y, Li X, Ren L, Zhao J, Hu Y et al (2020) Clinical features of patients infected with 2019 novel coronavirus in Wuhan. China Lancet 395(10223):497-506. https://doi. org/10.1016/S0140-6736(20)30183-5

Li Y, Li M, Wang M, Zhou Y, Chang J, Xian Y et al (2020) Acute cerebrovascular disease following COVID-19: a single center, retrospective, observational study. Stroke Vasc Neurol 5(3):279-284. https://doi.org/10.1136/svn-2020-000431

Lingeswaran M, Goyal T, Ghosh R, Suri S, Mitra P, Misra S et al (2020) Inflammation, immunity and immunogenetics in COVID-19: a narrative review. Indian J Clin Biochem 35(3):260-273. https://doi.org/10.1007/s12291-020-00897-3

Mo P, Xing Y, Xiao Y, Deng L, Zhao Q, Wang H et al. (2020) Clinical characteristics of refractory COVID-19 pneumonia in Wuhan, China. Clin Infect Dis ciaa270. https://doi.org/10.1093/ $\mathrm{cid} / \mathrm{ciaa} 270$

Netland J, Meyerholz DK, Moore S, Cassell M, Perlman S (2008) Severe acute respiratory syndrome coronavirus infection causes neuronal death in the absence of encephalitis in mice transgenic for human ACE2. J Virol 82(15):7264-7275. https://doi. org/10.1128/JVI.00737-08

Shahid Z, Kalayanamitra R, McClafferty B, Kepko D, Ramgobin D, Patel R et al (2020) COVID-19 and older adults: what we know. J Am Geriatr Soc 68(5):926-929. https://doi.org/10.1111/ jgs. 16472

Tang N, Li D, Wang X, Sun Z (2020) Abnormal coagulation parameters are associated with poor prognosis in patients with novel coronavirus pneumonia. J Thromb Haemost 18(4):844-847. https ://doi.org/10.1111/jth.14768

van Wamelen DJ, Leta V, Johnson J, Ocampo CL, Podlewska AM, Rukavina K et al (2020) Drooling in Parkinson's Disease: prevalence and progression from the non-motor international longitudinal study. Dysphagia 35(6):955-961. https://doi.org/10.1007/ s00455-020-10102-5

Wang D, Hu B, Hu C, Zhu F, Liu X, Zhang J et al (2020) Clinical characteristics of 138 hospitalized patients with 2019 novel coronavirus-infected pneumonia in Wuhan. China. JAMA 323(11):10611069. https://doi.org/10.1001/jama.2020.1585

WHO (2020) Clinical management of severe acute respiratory infection when novel coronavirus $(2019-\mathrm{nCoV})$ infection is suspected: interim guidance. https://www.who.int/publications-detail/clini cal-managementof-severe-acute-respiratory-infection-when-novel -coronavirus-(ncov)-infection-is-suspected

World Medical Association Declaration of Helsinki Ethical Principles for Medical Research Involving Human Subjects (2013) JAMA JAMA 310(20):2191-2194. https://doi.org/10.1001/ jama.2013.281053

Xu H, Zhong L, Deng J, Peng J, Dan H, Zeng X et al (2020) High expression of ACE2 receptor of 2019-nCoV on the epithelial cells of oral mucosa. Int J Oral Sci 12(1):8. https://doi.org/10.1038/ s41368-020-0074-x

Yang X, Yu Y, Xu J, Shu H, Xia J, Liu H et al (2020a) Clinical course and outcomes of critically ill patients with SARS-CoV-2 pneumonia in Wuhan, China: a single-centered, retrospective, observational study. Lancet Respir Med 8(5):475-481. https://doi. org/10.1016/S2213-2600(20)30079-5 
Yang X, Yu Y, Xu J, Shu H, Xia J, Liu H et al (2020b) Clinical course and outcomes of critically ill patients with SARS-CoV-2 pneumonia in Wuhan, China: a single-centered, retrospective, observational study. Lancet Respir Med 8(5):475-481. https://doi. org/10.1016/S2213-2600(20)30079-5

Zhu N, Zhang D, Wang W, Li X, Yang B, Song J et al (2020) A novel coronavirus from patients with pneumonia in China, 2019. N Engl J Med 382(8):727-733. https://doi.org/10.1056/NEJMoa2001017
Publisher's Note Springer Nature remains neutral with regard to jurisdictional claims in published maps and institutional affiliations. 